\title{
Um estudo sobre o desenvolvimento colaborativo de uma aplicação mobile para cadastro de vítimas de desastres naturais
}

\author{
A study on collaborative development of a mobile application for registration of natural disaster victims \\ Anrafel Fernandes Pereira*», Leonardo Lima Procópio $*$, Aline Miranda Marques*, Flávio Duarte \\ Pinto $^{\ddagger}$
}

Como citar esse artigo. Pereira AF, Procópio LL, Marques AM, Pinto Flávio Duarte. Um estudo sobre o desenvolvimento colaborativo de uma aplicação mobile para cadastro de vítimas de desastres naturais. Revista Eletrônica Teccen. 2016 Jan./Jun.; 09 (1): 26-34.

\begin{abstract}
Resumo
Buscar soluções para cenários de desastres naturais não é uma atividade trivial. Entretanto, com o apoio da comunidade, soluções simples e inteligentes podem surgir para apoiar ações necessárias em situações de emergência. O desenvolvimento de software tem migrado da maneira tradicional para um novo patamar, por exemplo, através do desenvolvimento colaborativo por times ou pessoas dispersas geograficamente. Neste artigo é investigado como ferramentas tradicionais de comunicação, gerenciamentos de atividades e de configuração podem apoiar o desenvolvimento colaborativo por uma comunidade virtual de desenvolvedores para auxiliar famílias vítimas de desastres naturais, ressaltando os principais aspectos de colaboração.
\end{abstract}

Palavras-Chave: Desenvolvimento Colaborativo; Desastres naturais; Comunidade de desenvolvimento; Aplicação móvel.

\begin{abstract}
A search solution for natural disaster scenarios is not a trivial activity. However, with community support, simple and intelligent solutions can come to support actions required in emergency situations. Software development has migrated from the traditional way to a new level, for example, through the collaborative development by teams or geographically dispersed people. This paper investigated as traditional communication tools, activity management and configuration can support the collaborative development of a virtual community of developers to assist victims of natural disasters families, highlighting the main aspects of collaboration.

Keywords: Collaborative Development; Natural disasters; Development community; Mobile application.
\end{abstract}

\section{Introdução}

Percebe-se com frequência a dificuldade de uma cidade, estado ou país conseguir resistir à ameaça de desastres naturais, mesmo sendo ameaçados em diferentes níveis, por exemplo, enchentes, maremotos, terremotos, entre outras. Diante disso, cada vez mais governantes e pesquisadores tem buscado mecanismos para minimizar as perdas e os danos oriundos destes tipos de eventos para a sociedade em geral, conforme pode ser visto nos trabalhos de Wattegama (2007), Lage et al. (2011) e De Faria Cordeiro et al. (2014). Neste contexto, a busca por soluções através de ferramentas computacionais tem se mostrado uma alternativa de sucesso, já que a preparação para catástrofes não é mais uma escolha, mas sim um caminho obrigatório, independentemente de onde se vive (De Faria Cordeiro et al., 2014).

Buscar soluções paracenários de desastres naturais não é uma atividade trivial. Isso pode ser percebido em vários trabalhos na literatura que exploram este contexto, conforme pode ser visto em Nascimento et al. (2014) e Dos Santos et al. (2014). Entretanto, com o apoio da comunidade, soluções simples e "inteligentes" podem surgir como forma de apoiar e facilitar os serviços e ações necessários diante de situações de emergência. $\mathrm{O}$ conceito de comunidade é costumeiramente usado para descrever um conjunto de pessoas em uma determinada área geográfica. Isso inclui ainda, a ideia de que esse conjunto possui uma estrutura social, onde existe algum tipo de relacionamento entre essas pessoas. Além disso, pode existir um espírito compartilhado entre os membros da comunidade e um sentimento de pertencer ao grupo (Pimentel \& Fuks, 2011).

Atualmente, a tecnologia da informação, principalmente a internet tem revolucionado o relacionamento humano. Através dela, tem-se aberto novas formas de intercâmbio de informações, de forma interativa, assíncrona ou síncrona, com significante intimidade mesmo que sem proximidade física. Com

\footnotetext{
Afiliação dos autores: †Universidade Severino Sombra, Vassouras-RJ, Brasil.

\$ FAETEC - Fundação de Apoio à Escola Técnica do Estado do Rio de Janeiro, Três Rios-RJ, Brasil.

$\S$ Universidade Federal do Rio de Janeiro, Rio de Janeiro-RJ, Brasil.

* Endereço para correspondência: Universidade Severino Sombra, Av. Exped. Oswaldo de Almeida Ramos, 280 - Centro - Vassouras, RJ - CEP 27700-000.

E-mail: anrafel@live.com
} 
isso, além das aplicações tradicionais, a internet abre outros canais de comunicação que permitem a interação simultânea de dezenas de pessoas. A partir daí um novo conceito de comunidade emerge como comunidades virtuais. Uma comunidade virtual pode ser definida como um conjunto de pessoas engajadas em discussões, interagindo em um ambiente virtual, por um tempo que é suficiente para o estabelecimento de relacionamentos pessoais (Pimentel \& Fuks, 2011). Através dela, novas ferramentas e soluções para diversos problemas enfrentados pela população de uma cidade, estado ou país, podem ser desenvolvidas, principalmente quando se trata de uma comunidade virtual de desenvolvedores.

Há tempo que a atividade de desenvolvimento de software tem atingido outros patamares, como por exemplo, o desenvolvimento colaborativo de software por times ou pessoas dispersas geograficamente, conforme é evidenciado em Audy \& Prikladnicki (2008) e Lopes (2004). Isso tem sido possível graças ao avanço tecnológico dos últimos tempos e principalmente devido ao grande número de ferramentas para apoiar o desenvolvimento de softwares neste cenário (Wolf \& Da Silva, 2014). O desenvolvimento colaborativo de software tem possibilitado ainda, que soluções possam ser retiradas do papel e aplicadas em situações reais e de variados contextos. Em algumas vezes, são desenvolvidos por programadores ou pessoas interessadas em tecnologia, dispersas geograficamente. $\mathrm{O}$ que torna ainda mais evidente a visão colaborativa de desenvolvimento de software, já que hoje o software tornou-se vital para os negócios de todos os tipos de organizações.

Em relação aos recursos tecnológicos, cada vez mais as potencialidades das redes e dos dispositivos móveis vem sendo exploradas no sentido de fornecerem um vasto conjunto de serviços e aplicações dos mais diversostipos, podemserconhecidas emalguns trabalhos, tais como, Souza \& Kushchu (2005), Education (2010) e Allen, Graupera \& Lundrigan (2010), por exemplo. Entre elas, podem-se citar as aplicações que oferecem apoio aos usuários em situações de emergência, foco deste trabalho científico.

Este trabalho está inserido em um contexto específico de uma situação de desastre ocorrida na região Centro Sul Fluminense do estado do Rio de Janeiro, no final do mês de Janeiro de 2016. De acordo com FEMA (2010) a ajuda inicial às vítimas, quase nunca chega num tempo inferior a 48 horas. Sendo assim, é fundamental criar mecanismos de auto-organização e autoproteção entre as famílias afetadas, principalmente para auxiliálas nas primeiras horas após uma catástrofe. Para isso, foi desenvolvida, de forma colaborativa, uma aplicação mobile, conhecida como Relpio, com o objetivo de apoiar as famílias vítimas da catástrofe.

Este artigo busca responder a seguinte questão de pesquisa: "Como ferramentas tradicionais de comunicação, gerenciamento de tarefas e gerenciamento de configuração, podem apoiar uma comunidade virtual durante a atividade de desenvolvimento colaborativo de uma aplicação para cadastro de vítimas de desastres naturais?". Tem como objetivo discutir o desenvolvimento de software enquanto atividade colaborativa, ressaltando os principais aspectos de colaboração neste contexto. Na seção 2 os trabalhos relacionados com este trabalho de pesquisa, são apresentados. A seção 3 apresenta o desenvolvimento do aplicativo Relpio, ressaltando os processos de engenharia de software envolvidos nesta atividade. $\mathrm{Na}$ seção 4, é apresentada a metodologia do estudo de caso realizado no trabalho, destacando também, os principais resultados e evidências obtidos com a realização do estudo. Por fim, a seção 5 apresenta as considerações finais deste trabalho.

\section{Trabalhos Relacionados}

Diferentes trabalhos encontrados na literatura estão inseridos no contexto de situações de emergências (De Faria Cordeiro et al., 2014; Lage et al., 2011). Destes, alguns trabalhos exploram aspectos relacionados com a colaboração de maneira que possam auxiliar a minimização e/ou resolução de problemas em algum cenário de situação de emergência (Nascimento et al. 2014; dos Santos et al., 2014) ).

Em Nascimento et al. (2014), os autores têm a preocupação em oferecer aos órgãos responsáveis uma abordagem para apoiá-los na seleção e visualização colaborativa de informações de situações de emergência. Para isso, um dashboard personalizado é apresentado para os gestores de forma que ele consiga configurar os tipos de informações relevantes sobre uma determinada situação de emergência. Neste artigo, o foco está em um primeiro estágio, pois se concentra apenas na captura das informações oferecidas pelo usuário ou por um agente de um órgão responsável pelo apoio à famílias vítimas de desastres naturais, através do aplicativo Relpio.

Dos Santos et al. (2014) abordam o desenvolvimento de famílias de sistemas colaborativos, baseado em uma Linha de Produto de Software, para apoiar a gestão de situações de risco de inundação. Neste trabalho, o interesse principal foi o de criar uma aplicação para apoiar famílias vítimas de inundações causadas pela cheia do Rio Paraíba do Sul em cidades do interior do estado do Rio de Janeiro. Entretanto, não foi o interesse do grupo, o desenvolvimento de uma aplicação colaborativa, como é apresentado em Dos Santos et al. (2014), mas o interesse de construir de forma colaborativa uma aplicação para apoiar pessoas inseridas neste contexto. Entretanto, o trabalho de Dos Santos et al. (2014) e este trabalho compartilham contextos relativamente semelhantes. 
O desenvolvimento colaborativo já foi tratado na literatura de Sistemas Colaborativos (Werner et al., 2003); Costa et al., 2008). Entretanto, estes trabalhos não abordam a atividade de desenvolvimento de software realizada por uma comunidade virtual de desenvolvedores, mas sim realizada por uma equipe de desenvolvimento tradicional. No trabalho de Werner et al. (2003) os autores têm a preocupação com o desenvolvimento colaborativo de componentes de software, enfatizando a importância dos participantes de uma equipe de desenvolvedores de colaborarem para compartilhar os seus conhecimentos sobre a identificação, especificação, construção e reutilização de um determinado componente. Para isso, apresentam um ambiente identificado como OdysseyShare para apoiar a colaboração da equipe durante a realização das suas atividades. Neste trabalho, o foco está na avaliação das ferramentas que estão sendo utilizadas pelos membros da comunidade virtual de desenvolvedores ( $G D G$ Três Rios) para desenvolverem o aplicativo móvel Relpio, de forma a responder a questão de pesquisa deste artigo.

Em Costa et al. (2008), a engenharia de software é explorada como atividade colaborativa. Para isso, os autores apresentam uma ferramenta identificada como RaisAware para auxiliar a atividade de desenvolvimento de software baseada em análises de dependências. Neste trabalho, a atividade de engenharia de software é detalhada na seção 3, de maneira a apresentar a metodologia utilizada pelo grupo, bem como as ferramentas adotadas para a construção do produto de software que é apresentado na seção de avaliação deste artigo.

\section{Desenvolvimento do Aplicativo Relpio}

Nesta seção é apresentado o desenvolvimento do aplicativo mobile, Relpio. O objetivo principal da aplicação é servir como um mecanismo para auxiliar que famílias vítimas de uma catástrofe natural possam realizar o seu cadastro solicitando ajuda aos órgãos responsáveis, e ainda, apresentar os principais pontos de apoio para recebimento de doações, de forma que pessoas interessadas em ajudar estas famílias possam encontrar os locais apropriados para realizarem sua doação.

A iniciativa para o desenvolvimento deste aplicativo mobile surgiu a partir do problema identificado na cidade de Paraíba do Sul / RJ, onde residem alguns dos membros da comunidade virtual de desenvolvedores, conhecida como Google Developers Group. O Google Developers Group - GDG Três Rios é uma comunidade com aproximadamente doze participantes mais ativos. O grupo se reúne virtualmente, através de videoconferências e presencialmente, alguns dias do mês para trocarem experiências sobre desenvolvimento e desenvolverem projetos de interesse comuns aos participantes. Portanto, o grupo pode ser considerado como uma comunidade virtual de desenvolvimento de software.

A definição do contexto em que o software atuaria e quais seriam os papéis desempenhados por cada membro do grupo foi definindo o fluxo de colaboração entre as pessoas. Após esta etapa, foram especificados os processos necessários que deveriam ser realizados para que os produtos de software pudessem ser entregues. Alguns exemplos dos produtos definidos foram: a descrição dos requisitos funcionais e não funcionais, prototipação, o código e os casos de teste, que serão apresentados nas subseções a seguir.

\section{Identificações dos Requisitos}

Existem diversas técnicas de identificação de requisitos, e que são adequadas a diferentes situações. Entre estas técnicas está a especificação de cenários (Bronze et al., 2014). Trata-se de uma abordagem informal, mas através dela, é possível levar as pessoas a imaginarem o comportamento de um sistema, e ainda, poderem comentar acerca do seu comportamento e da interação que esperam ter com ele. Para ilustrar o cenário no qual o aplicativo mobile Relpio pode ser

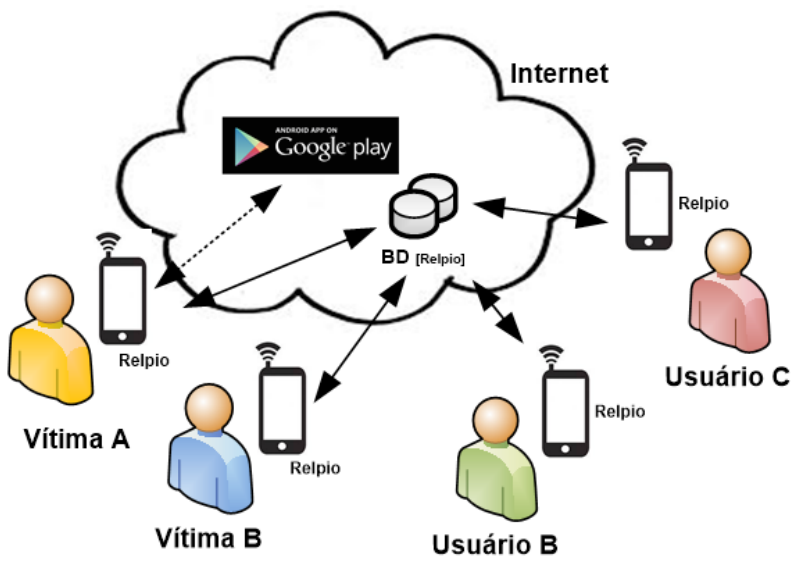

Figura 1. Visão Geral da Aplicação Relpio. Fonte: o autor.

utilizado, a Figura 1 é apresentada.

A especificação de um cenário foi a forma escolhida para realizar a identificação dos requisitos necessários para desenvolver a ferramenta. A partir daí, foi desenvolvida a prototipação, que será apresentada na subseção seguinte.

\section{Prototipagem}

Foi desenvolvido um protótipo da aplicação para que os desenvolvedores pudessem visualizar algumas funcionalidades do aplicativo, o que facilitou o entendimento das funcionalidades do sistema, já que 
os desenvolvedores se encontravam geograficamente dispersos. A Figura 2 ilustra o protótipo desenvolvido. $\mathrm{O}$ protótipo possibilitou o refinamento de alguns requisitos já identificados e a identificação de novos requisitos. A prototipação possibilita ao desenvolvedor criar um modelo de aplicativo que será implementado, retratando como será a interação do usuário com o aplicativo e quais funcionalidades deverão ser implementadas e aperfeiçoadas (Audy e Prikladnick, 2008).

As principais funcionalidades do aplicativo são o cadastro de vítimas da catástrofe, o acesso aos pontos de apoio e o acesso das informações de cadastro pelos órgãos competentes e responsáveis para auxiliar as vítimas. $\mathrm{O}$ cadastro pode ser feito pelas próprias vítimas ou por algum órgão responsável no momento de abordagem às famílias, como por exemplo, assistentes sociais, agentes do bombeiro e da defesa civil, dentre outros. Depois de cadastradas, as informações são disponibilizadas apenas para os órgãos competentes e responsáveis para auxiliar as vítimas. Outra funcionalidade destacada é o acesso aos pontos de apoio por pessoas que buscam ajudar as vítimas afetadas pelo evento.

Todos os cadastros realizados são armazenados em um banco de dados na nuvem. Para isso, a conexão com a internet é fundamental para utilização do aplicativo. Através dos cenários definidos, os requisitos funcionais e não funcionais e a prototipação avaliada, os próximos passos foram executados, discutidos na subseção a seguir.

\section{Aspectos de Implementação}

Adotar padrões de desenvolvimento permite que as equipes se mantenham alinhadas e facilita ainda a manutenção dos sistemas, pois mantém os códigos e os artefatos gerados uniformes. Outro ponto importante é a adoção de frameworks, pois eles já trazem funcionalidades importantes e mais robustas para as linguagens de programação, o que acaba diminuindo esforços de tempo e custo no processo de desenvolvimento (Santos, Feller \& Motta, 2014).

Para atender o requisito não funcional de disponibilidade da aplicação para qualquer plataforma móvel, a comunidade optou pelo desenvolvimento híbrido de aplicativos. O desenvolvimento de aplicações mobile em ambiente nativo para cada plataforma do mercado requer uma demanda muito grande de tempo, esforço e custo, o que acaba impactando diretamente no produto final (Procópio \& Pereira, 2015). O desenvolvimento de aplicativos mobile multiplataforma por outro lado, permite que o código seja implementado uma única vez, podendo ser distribuída para as diferentes plataformas existentes no mercado com menor esforço de tempo e custo.

Sendo assim, as linguagens utilizadas para o desenvolvimento da aplicação foram: HTML (HyperText Markup Language), CSS (Cascading Style Sheets) e JavaScript, visto que o grupo já tinha experiência e também devido à baixa curva de aprendizagem da mesma. O Framework7 (disponível em: http://framework7.io/) foi o escolhido para apoiar o desenvolvimento, especialmente por ser open source e possibilitar que a aplicação gerada pudesse ser bem parecida com aplicações nativas para as plataformas Android e IOS. Outro framework adotado pelo grupo foi o Cordova, responsável por empacotar todo o código e gerar as versões dos aplicativos compatíveis com as principais plataformas do mercado. Entretanto, a aplicação foi disponibilizada apenas para dispositivos com o sistema operacional Android, devido a
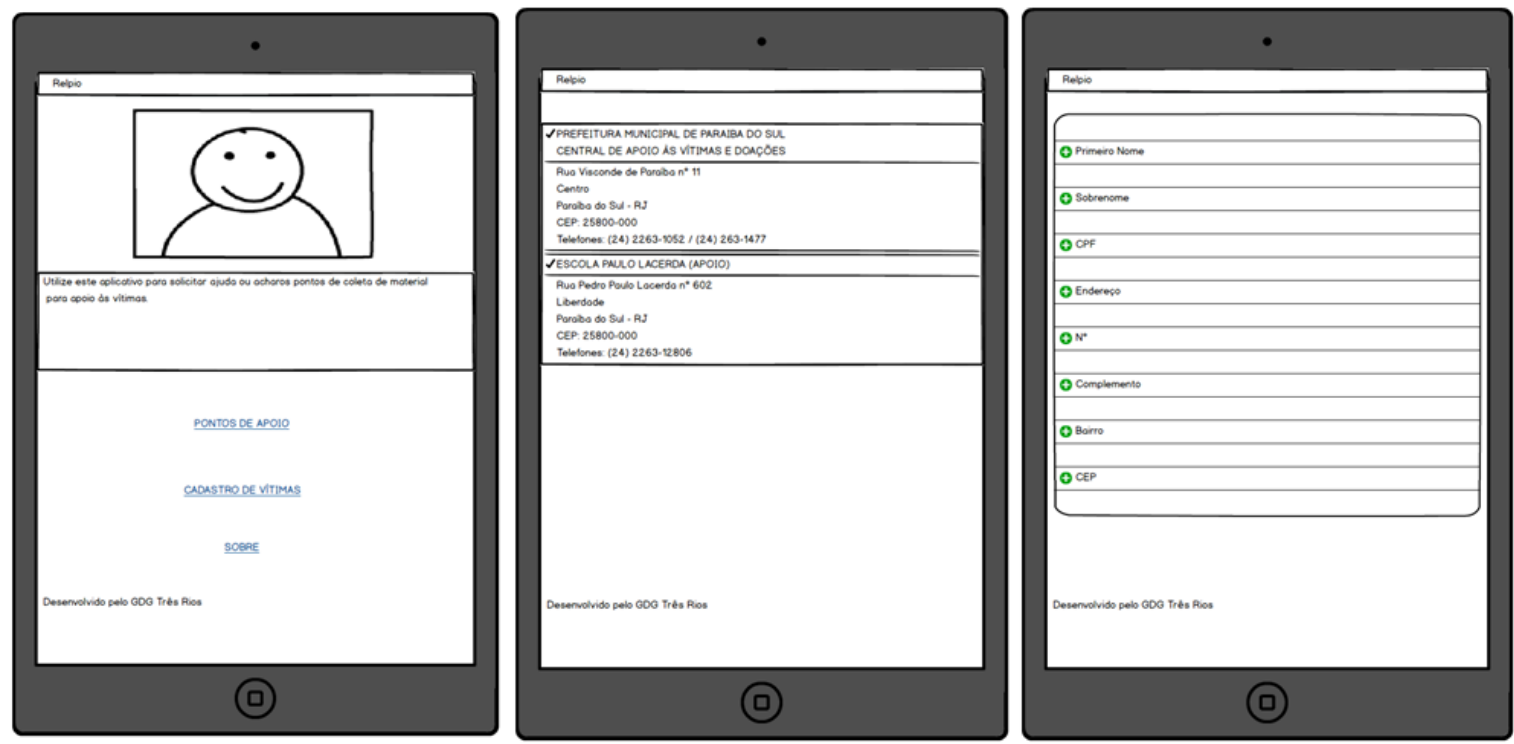

Figura 2. Protótipo de Telas da Aplicação Relpio.

Fonte: o autor. 
comunidade já possuir licença para a disponibilização de aplicativos para esta plataforma.

Para agilizar o início do projeto de desenvolvimento do aplicativo Relpio foi disponibilizado para os desenvolvedores um esqueleto inicial contendo os arquivos necessários para começalo no padrão especificado, utilizando o Framework7. O modelo disponibilizado já continha as configurações comuns e padrão do projeto, exemplos de uso de componentes de interface e ainda as chamadas dos componentes padrões, por exemplo.

No cenário deste trabalho fica evidente que houve a necessidade de existir ambientes distintos para o desenvolvimento do projeto. Com isso, ambientes específicos precisaram ser configurados para que cada membro pudesse realizar a sua atividade. Isso foi importante devido a alguns problemas que aconteceram, como por exemplo, a realização de alteração e inclusão de dados diretamente no banco de dados da aplicação, alterações realizadas diretamente na estrutura do banco de dados que estava disponível em um servidor web externo para todos os membros da comunidade, o que acabava causando desatualização do ambiente de desenvolvimento de outro membro, entre outros.

Em relação aos testes, verificações e validações funcionais na aplicação eram realizadas constantemente por um dos membros, como forma de garantir a qualidade do produto entregue. Além disto, testes unitários das funcionalidades da aplicação também foram realizados. À medida que falhas e melhorias foram sendo observadas, ajustes foram realizados de forma a preparar a aplicação para ser disponibilizada.

\section{Estudo de Caso}

A metodologia empregada para a avaliação deste trabalho foi o estudo de caso. Este método é recomendado quando a pesquisa necessita de uma investigação empírica dos fenômenos dentro de um contexto real (Yin, 2009). Neste caso, o contexto diz respeito ao desenvolvimento colaborativo de um aplicativo para apoiar famílias vítimas de desastres naturais ocorrida na região Centro Sul Fluminense do estado do Rio de Janeiro, no final do mês de Janeiro de 2016. Para este estudo, foram observadas questões relacionadas aos aspectos de colaboração, baseado no modelo 3C de Colaboração (Fuks et al., 2007), durante a atividade de desenvolvimento de software por uma comunidade virtual de desenvolvedores. O Modelo 3C de Colaboração é frequentemente usado pela literatura para classificar os sistemas colaborativos, destacando três classes de funcionalidades que uma aplicação colaborativa deve dar suporte, são elas: Coordenação, Cooperação e Comunicação.

Aquestão de pesquisa formulada para este estudo foi: Como ferramentas tradicionais de comunicação, gerenciamento de tarefas e gerenciamento de configuração podem apoiar uma comunidade virtual durante a atividade de desenvolvimento colaborativo de uma aplicação para cadastro de vítimas de desastres naturais?

A dimensão observada foi realizada através da seguinte variável dependente: a eficácia do processo de desenvolvimento adotado pelos participantes do grupo durante o desenvolvimento do aplicativo Relpio em relação às ferramentas utilizadas para apoiar o desenvolvimento colaborativo, que tem como objetivo principal cumprir um papel de aspecto social nas cidades onde foi inserido.

A respeito da caracterização do grupo, todos os membros participantes deste estudo já eram desenvolvedores ou tinham alguma experiência com desenvolvimento. Entre eles, havia participantes como formação técnica, graduação, especialização e mestrado em alguma área relacionada a informática.

Para apoiar o desenvolvimento da aplicação proposta buscou-se suporte em ferramentas colaborativas que pudessem auxiliar nesta atividade. Quando se tem muitas pessoas trabalhando em um mesmo sistema ou aplicação, torna-se indispensável o controle das alterações realizadas no código, e até mesmo em outros artefatos gerados pela atividade de desenvolvimento. Para isso, a ferramenta GitHub foi utilizada para disponibilizar o código para que todos os membros desenvolvedores pudessem ter acesso aos artefatos. O versionamento do código-fonte fornece aos participantes a oportunidade de controlar os responsáveis por cada mudança no artefato, e ainda dividir as entregas dos produtos. O papel de uma ferramenta de gerência de configuração neste contexto é importante justamente para apoiar a coordenação e cooperação necessária para manter a integridade dos produtos de softwares.

Todo o desenvolvimento do projeto foi realizado de maneira distribuída. Os membros envolvidos neste cenário trabalharam de locais remotos, estando geograficamente dispersos, em bairros e cidades distintas, nenhum encontro presencial foi realizado. A Figura 3 ilustra os níveis de dispersão dos membros do grupo, bem como de todos os participantes envolvidos neste trabalho. Apresenta também a relação entre os membros, focando na estrutura de comunicação durante o processo de desenvolvimento do aplicativo, envolvendo essencialmente as fases de levantamento de requisitos (membros do grupo do bairro A-C), coordenação das tarefas desempenhadas por cada participante (membros do grupo do bairro B-A), atividades de desenvolvimento do aplicativo (membros do grupo do bairros A-A, B-C, B-F e B-B), além das atividades de teste, desempenhadas por membros do grupo do bairro B-B. A maior parte da comunicação 


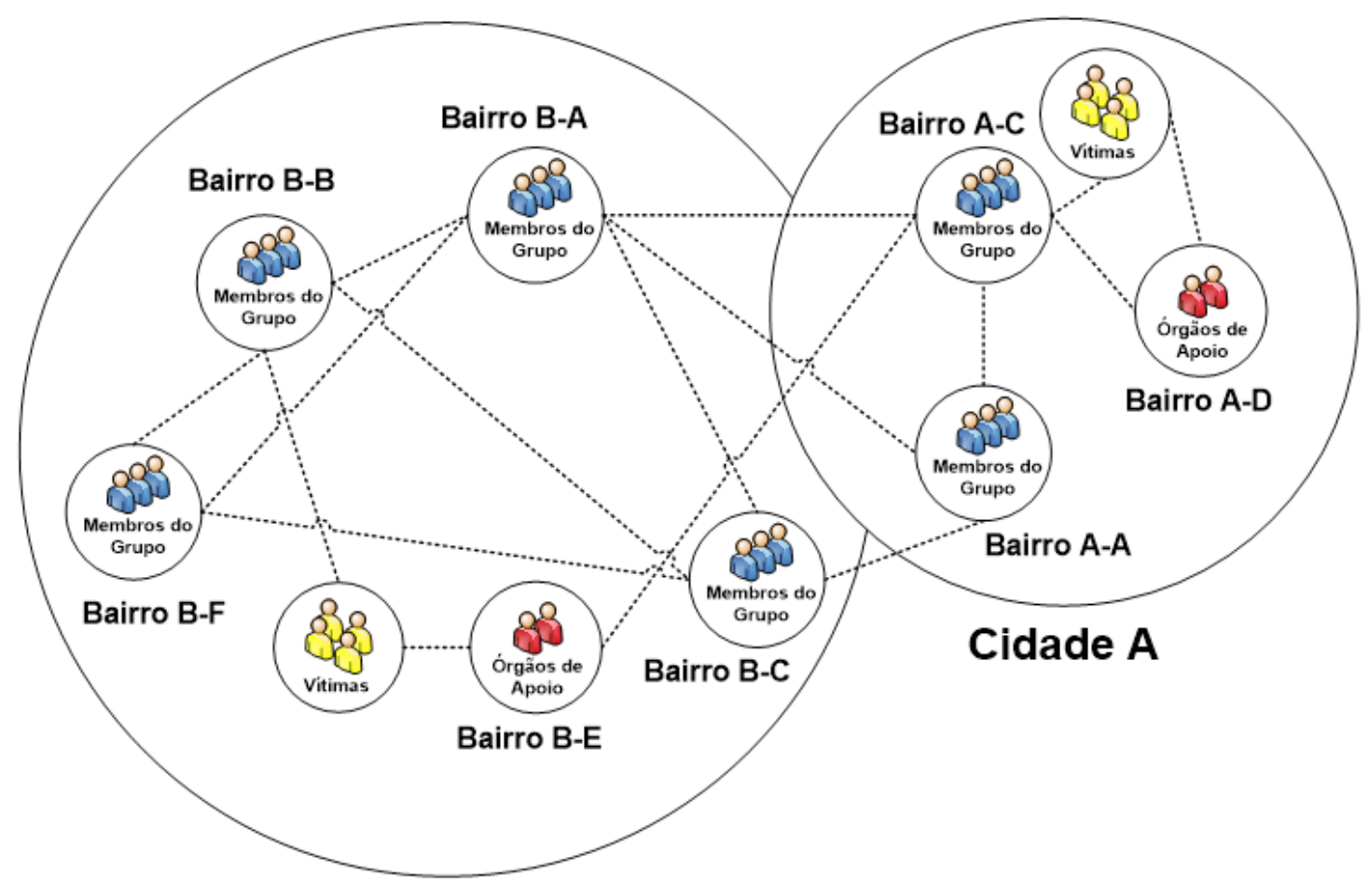

Cidade B

Figura 3. Níveis de Dispersão do Grupo GDG Três Rios.

Fonte: o autor.

entre os membros aconteceu através da ferramenta de hangout da Google e Whatsapp.

Mecanismos para apoiar a coordenação das atividades e dos membros envolvidos também foram necessários. O roteiro com as atividades necessárias foi organizado e distribuído inicialmente através de um e-mail para cada membro do grupo. Depois, para que os demais membros pudessem conhecer de fato a atividade que estava sendo desempenhada por cada participante da comunidade de desenvolvimento, uma ferramenta que pudesse apoiar a coordenação foi escolhida, sendo ela a ferramenta Trello. Através dela, pode-se perceber quais eram realmente os participantes envolvidos em cada etapa e em que eles estavam trabalhando.

\section{Resultados do Estudo}

Os dados coletados e utilizados neste estudo foram obtidos através de históricos de conversas e atividades registradas pelo grupo e através de observações diretas, visto que alguns dos autores deste trabalho participaram ativamente do projeto. Foram coletados os dados de e-mails, tarefas registradas em uma ferramenta de gerenciamento de atividades (Trello) e histórico de mensagens através de mecanismos de comunicação (Hangout e Whatsapp). Estes dados foram coletados no período de fevereiro de 2016 e utilizados como fonte para o desenvolvimento deste trabalho de pesquisa.

As tarefas designadas para cada subject no estudo realizado dizia respeito às atividades que foram especificadas na ferramenta de gerenciamento de tarefas - Trello (https://trello.com/), portanto eram conhecidas por todos os participantes do estudo. Com base no modelo $3 \mathrm{C}$ de Colaboração (Fuks et al., 2007), a ferramenta Trello pode ser caracterizada como uma ferramenta de Coordenação. Uma lista dos membros com as respectivas atividades desenvolvidas estava visível para todos os participantes, gerando a oportunidade de coordenação de uma ou várias atividades. Um participante membro do bairro B-A estava assumindo o papel de coordenador das atividades.

A ferramenta GitHub (https://github.com/) foi utilizada como um template inicial para a aplicação desenvolvida. Esta ferramenta é utilizada para apoiar o gerenciamento de configuração em ambiente de desenvolvimento de software. Assim, cada subject que estava responsável pela tarefa de desenvolvimento pode cooperar no projeto de maneira que pudesse conhecer também o trabalho dos demais participantes. O projeto passa a ser desenvolvido em uma cópia local para cada um dos participantes, e a medida que as tarefas pré-definidas foram sendo concluídas, eram submetidas ao código original no servidor do Github, passando a estar disponível a todos os envolvidos no projeto. Por exemplo, rotinas que já estavam sendo feitas, protótipo que havia sido criado, modelo do banco de dados, entre outros. Baseado no modelo 3C de colaboração, a ferramenta pode ser caracterizada como um elemento de Cooperação.

O elemento de Comunicação do modelo 3C 
de Colaboração pode ser verificado essencialmente em 3 ferramentas que foram utilizadas: (i) correio eletrônico, o tradicional e-mail (ii) Hangout (https:// hangouts.google.com/) - ferramenta de comunicação da Google; e (iii) Whatsapp (https://www.whatsapp. $\mathrm{com} /$ ). O suporte à comunicação foi o um meio para que os membros do grupo pudessem trocar experiências e/ou esclarecerem possíveis dúvidas sobre um ponto específico das atividades que estavam sendo realizadas. Esta atividade contribuiu com o trabalho dos demais participantes mantendoos conscientes sobre as ações realizadas pelo grupo, e permitindo uma interação rápida em qualquer fase do projeto, onde cada participante poderia opinar ou colocar em questão algum ponto do projeto. A ausência de um mecanismo como este, faz com que os membros trabalhem e tomem decisões amparadas em seu próprio conhecimento e experiência. Além disso, impossibilita que interações espontâneas entre os participantes sejam iniciadas e dificulta a colaboração entre eles. Sendo um dos desafios relacionados ao desenvolvimento distribuído de software (Audy \& Prikladnicki, 2008).

Em relação ao aspecto de comunicação, foi observado que os membros conseguiram trocar informações de forma clara e objetiva. Questões que geravam dúvidas na realização de uma atividade eram discutidas e apoiadas através da exploração de recursos extras, como por exemplo, imagens e fotografias que pudessem deixar claro a explicação de algum ponto. Assim, acredita-se que as ferramentas de comunicação utilizadas neste processo contribuíram de maneira satisfatória para o objetivo do grupo. Entretanto, vale ressaltar que mecanismos que ofereça suporte a Awareness sempre são necessários para contextualizar os membros da equipe sobre o estado da atividade dos demais membros do grupo e ainda apoiar a coordenação deles.

Outro elemento importante e também presente no modelo 3C de Colaboração é a percepção ou Awareness (Fuks et al., 2007). Segundo Audy \& Prikladnicki (2008) durante o desenvolvimento de software é importante que se tenha consciência do que está acontecendo, quem está realizando determinada tarefa, ou ainda, onde ela está acontecendo. Tudo isso, permite que os membros de um grupo possam ampliar a sua colaboração quando se tem estas informações. Neste estudo de caso os membros do grupo puderam ter consciência das atividades e tarefas que estavam sendo realizadas pelos demais membros, através das informações disponíveis nas ferramentas que foram empregadas para a realização da atividade de desenvolvimento do aplicativo.

O tempo total para a concepção das atividades que envolveram o desenvolvimento do aplicativo foi de aproximadamente 36 horas, não sequenciais, visto que se tratava de um aplicativo de cadastro e disponibilização de alguns dados para visualização. Para ilustrar o relacionamento entre as ferramentas utilizadas e os elementos de colaboração identificados no contexto deste estudo, segundo o modelo $3 \mathrm{C}$ de colaboração, a Figura 4 é apresentada.

Conforme pode ser observado através da Figura 4 , o resultado final da atividade de desenvolvimento foi o aplicativo Relpio, disponível através do endereço: https://play.google.com/store/apps/details?id=br. gdgtresrios.relpio\&hl=pt_BR. O aplicativo apoiou o cadastro de 102 vítimas da catástrofe e teve uma média de 45 downloads na loja oficial de aplicativos da Google. O Relpio foi utilizado por vítimas da enchente do rio Paraíba do Sul, órgãos responsáveis por apoio às famílias, e ainda por pessoas que buscavam formas de ajudar aos desabrigados e afetados pelo evento.

\section{Considerações Finais}

Este artigo teve como objetivo principal discutir o desenvolvimento de software enquanto atividade colaborativa, ressaltando os principais aspectos de colaboração no contexto de desenvolvimento colaborativo por uma comunidade virtual de desenvolvedores. Foi investigado, através de um estudo de caso, como a utilização de ferramentas tradicionais de comunicação, gerenciamento de tarefas e gerenciamento de configuração pode apoiar uma comunidade virtual durante a atividade de desenvolvimento colaborativo de um aplicativo de propósito social.

Através deste estudo, foi possível obter evidências sobre como o desenvolvimento de software tem migrado da maneira tradicional para um novo patamar, cheio de dificuldades e desafios para serem explorados, tais como: contextos diferentes para desenvolvimento, dispersão geográfica dos desenvolvedores, entre outros. Em relação às ferramentas utilizadas (e-mail, hangout, Whatsapp, Trello e GitHub) pode-se encontrar evidências sobre o apoio oferecido por elas para a realização das atividades propostas, identificando como os elementos de colaboração (Coordenação, Cooperação, Comunicação e Awareness) puderam ser observados e tratados no contexto deste trabalho.

Outro ponto importante de ser ressaltado é a importância das comunidades de desenvolvedores de software independentes no apoio ao desenvolvimento de soluções simples e "inteligentes" para a população em geral, de forma a minimizar "pequenos" problemas enfrentados pelas cidades, e ainda, agirem como cidadãos que exercem o papel de aplicar a tecnologia em prol da sociedade. 
Ferramentas de apoio ao desenvolvimento colaborativo

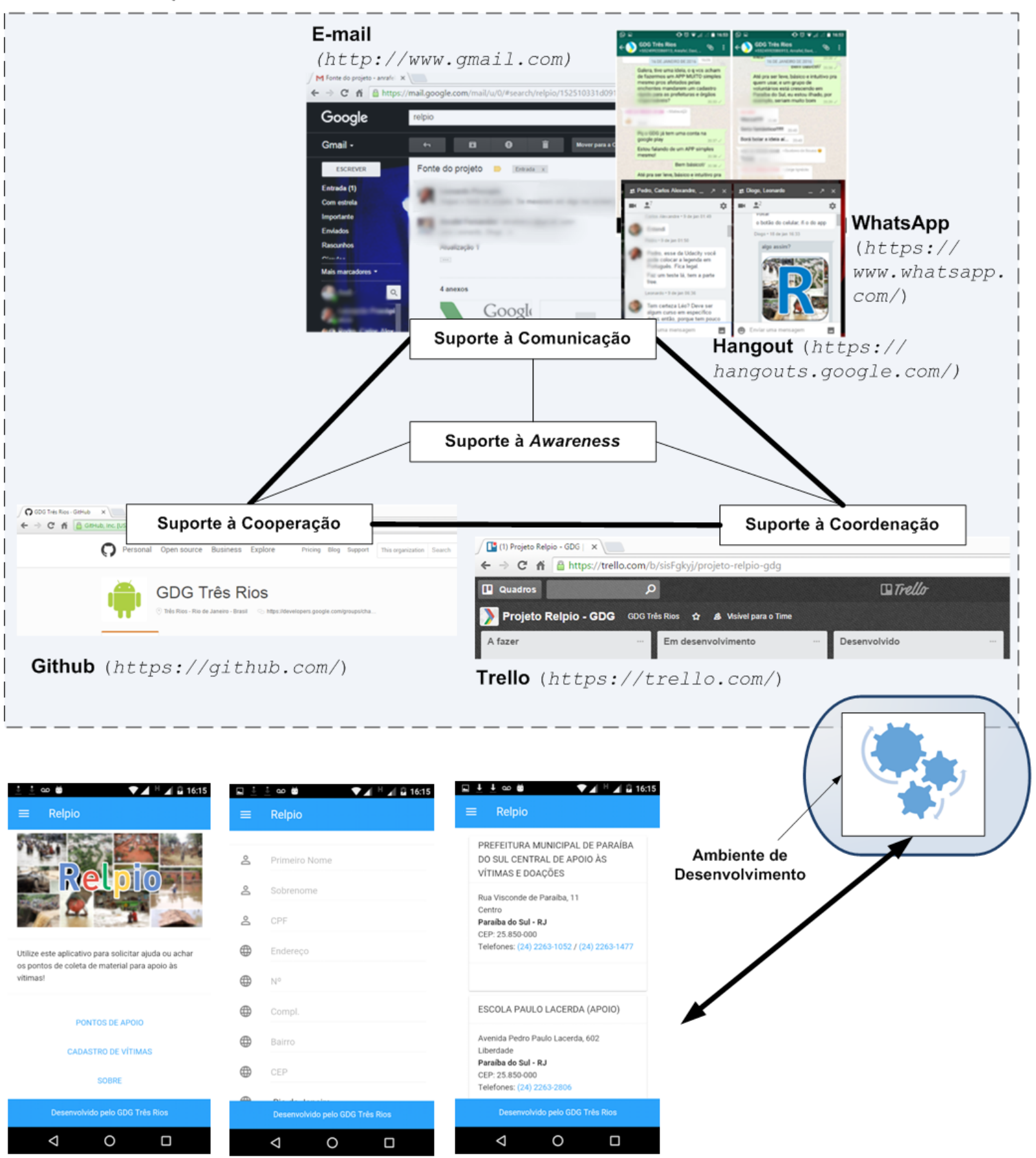

Relpio (https: //play.google.com/store/apps/ details?id=br.gdgtresrios.relpio\&hl=pt_BR)

Figura 4. Relacionamento entre as Ferramentas Utilizadas. Fonte: o autor.

\section{Referências}

Allen, S., Graupera, V., \& Lundrigan, L. (2010). Pro Smartphone CrossPlatform Development: iPhone, Blackberry, Windows Mobile and Android Development and Distribution. (1st ed). New York: Apress.

Audy, J. L. N.; Prikladnicki, R. (2008) Desenvolvimento distribuído de software: desenvolvimento de software com equipes distribuídas. Rio de Janeiro: Elsevier.

Bronze, R., Maia, F., Lima, W., \& Rocha, P. (2014). Levantamento de requisitos de software, uma análise comparativa. Sistemas de Informação \& Gestão de Tecnologia, n. 6, 2014.
Cordeiro, K.F.; Marino, T., Campos, M.L.M.; Borges, M.R.S. (2011) Use of Linked Data in the Design of Information Infrastructure for Collaborative Emergency Management System. International Conference on Computer Supported Cooperative Work in Design (CSCWD).

Costa, J. M., Feitosa, R. M., \& Souza, C. (2008). RaisAware: Uma ferramenta de auxílio à engenharia de software colaborativa baseada em análises de dependências. In Sistemas Colaborativos, 2008 (pp. 254-264). IEEE.

De Faria Cordeiro, Kelli; Campos, Maria Luiza Machado; Da Silva Borges, Marcos Roberto. (2014). Adaptive Integration of Information Supporting Decision Making: A Case on Humanitarian Logistic. Proceedings of ISCRAM 2014. 
Dos Santos Rocha, R., Degrossi, L. C., de Albuquerque, J. P., \& Horita, F. E. (2014). AGORA-PL: Uma Proposta para Desenvolvimento de Famílias de Sistemas Colaborativos baseados em VGI para a Gestão do Risco de Inundação. In Symposium on Collaborative Systems. October (Vol. 6, p. 9).

Education, U. S. D. O. (2010). Action Guide for Emergency Management At Institutions of Higher Education. Acesso em: 27/01/2016, from http://rems. ed.gov/docs/REMS_ActionGuide.pdf

FEMA, Developing and Maintaining Emergency Operations Plans: Comprehensive Preparedness Guide (CPG) 101. 2 v. 2010.

Fuks, H., Raposo, A., Gerosa, M.A., Pimentel, M. \& Lucena, C.J.P., (2007). "The 3C Collaboration Model". The Encyclopedia of E-Collaboration, Ned Kock (org), ISBN 978-1-59904-000-4, pp. 637-644, 2007.

Lage, B. B.; Borges, M. R. S.; Canós, J. H.; Vivacqua, A. S. (2011). Facilitating collaborative scenario creation to support emergency plan generation. In: Fifteenth International Conference on Computer Supported Cooperative Work in Design (CSCWD), Junho 2011, Lausanne, Switzerland. Proceedings. Estados Unidos: IEEE, 2011, p. 657-664.

Lopes, Leandro T. Um modelo de processo de engenharia de requisitos para ambientes de desenvolvimento distribuído de software. Dissertação (Mestrado) - Ciência da Computação, Universidade Católica do Rio Grande do Sul (PUCRS), 2004.

Nascimento, S.B., Borges, M.R.S, Vivacqua, S. A., (2014) Uma Abordagem para Seleção e Visualização Colaborativa de Informações em Situações de Emergência. In Symposium on Collaborative Systems.

Pimentel, M.; Fuks, H. (2011) "Sistemas Colaborativos". Rio de Janeiro - RJ: Elsevier-Campus-SBC, 2011.416 p.

Procópio, L. L.; Pereira, A. F. (2015). Buscando agilidade no desenvolvimento de aplicações Mobile através do desenvolvimento multiplataforma. In: XIV Encontro de Iniciação Científica - USS, 2015, Vassouras / RJ. Anais do XIV Encontro de Iniciação Científica. Vassouras: Editora USS, 2015. p. 173173.

Santos, H. P. D., Feller, N. J., \& Motta, T. S. (2014). Desenvolvimento colaborativo e integrado de sistemas: caso de sucesso no CPD-UFRGS. InWorkshop de Tecnologia da Informação e Comunicação das IFES (8.: 2014: Brasília).[Anais..]. Brasília: sn, 2014.

Santos, S. R. Um Modelo de Referência para Avaliação da Capacidade de Resposta das Organizações de Emergência. 2007. Dissertação (Mestrado em Informática) - Instituto de Matemática, Núcleo de Computação Eletrônica, Universidade Federal do Rio de Janeiro, Rio de Janeiro, 2007.

Souza, F., \& Kushchu, I. (2005). Mobile Disaster Management System Applications-Current Overview And Future Potential - Mlife conference \& exhibitions.

Wattegama, C (2007). ICT for disaster management, United Nations Development Programme-Asia-Pacific Development Information Programme (UNDP-APDIP).

Werner, C., Borges, M. R., Mattoso, M., Braga, R., Campos, F., Mangan, M., \& Vieira, V. (2003). OdysseyShare: Desenvolvimento colaborativo de componentes. IX Simpósio Brasileiro de Sistemas Multimídia e Web.

Wolf, Alexandre Stürmer; Da Silva, Maurício Severo. Ferramenta Para Gerenciamento E Apoio Ao Desenvolvimento Distribuído De Software. Destaques Acadêmicos, V. 5, N. 4, 2014.

YIN, R. K. (2009) Case Study Research: Design and Methods. 4 ed. Thousand Oaks, CA: Sage. 